\title{
A Blockchain Solution to Gun Control
}

\author{
Thomas F Heston $\mathrm{MD}^{1}$ \\ ${ }^{1}$ Department of Medical Education and Clinical Sciences, Washington State University, \\ Spokane, Washington USA
}

October 11, 2018

\begin{abstract}
Blockchain technology can be utilized to improve gun control without changing existing laws. Firearm related mortality is at epidemic levels in the United States and not only has a significant impact upon public health, it also creates a large financial burden. Suicide is the most common way guns kill. Through better gun tracking and improved screening of high risk individuals, this technological advance in distributed ledger technology will improve background checks on individuals and tracing of guns used in crimes.
\end{abstract}

\section{Introduction}

Blockchain technology can help reduce firearm related morbidity and mortality by improving background checks, gun registration, and decreasing economic pressures that increase interpersonal violence and suicides. In the US, firearm related deaths have reached epidemic levels. Suicides were the 10th leading cause of death and homicides the 17th leading cause of death in 2014, with the majority of these due to firearm injury [1]. The level of morbidity and mortality due to firearm injuries in the US is not showing a significant decline in spite of advances in medical care and technology. Death from firearms rightly has been recognized as a public health crisis [2].

A multi-pronged approach to reducing firearm related morbidity and mortality is necessary, given the limits on gun control legislation imposed by the 2nd Amendment to the US Constitution, which grants individuals the right to bear arms [3]. Existing legislation, designed to work within the bounds of the 2nd Amendment, has done little to reduce the staggering toll of gun violence [4]. More holistic approaches have shown some promise but implementation has been difficult. For example, the Violence Reduction Strategy (VRS) program implemented in Chicago in 2009 has had good success where it was able to be applied, reducing gun victimization by over $30 \%$. The success of the VRS program upon Chicago gun deaths was minimal, however, because it was only able to reach about $15 \%$ of the entire target population [5].

The approach suggested here is to apply blockchain technology holistically in a fashion specifically designed to reduce firearm related injuries. A primary component of this approach is to emphemeralize older, labor intensive approaches to reducing firearm deaths by applying a distributed ledger technology to background checks and gun tracking. Applying blockchain technology to gun control legislation will not only improve enforcement, it likely will also allow better legislation to be passed because it would address privacy issues which are important to gun rights advocates.

In addition, adopting blockchain protocols into the financial sector is also recommended because of the public health effects of a volatile economy. Although there is not a clear correlation globally, in the US, suicide is affected by economic conditions [6]. As the majority of firearm associated deaths are due to suicide, strategies to reduce precipitators of suicide such as unemployment and a poor business climate must be implemented. 
As such, blockchain applications in central banking should rightly be viewed as a potential way to decrease firearm violence by minimizing wide swings in economic conditions.

\section{Economic Impact of Gun Violence}

The primary economic impact of firearm related violence is years of potential life lost (YPLL), or in economic terms, opportunity costs. In 2015, the YPLL before age 65 due to firearm related deaths was 842,471 in the US. To put this number in perspective, the impact of fatal firearms injuries was more than three times greater than the impact of diabetes, and almost four times greater than cerebrovascular disease [7]. Furthermore, business recessions have also been shown to increase the suicide rate primarily among people that are in the prime working ages of 25 to 64 [6]. When all injuries are considered, over 100,000 Americans are killed or injured every year due to firearms [8].

Although a dollar amount cannot be placed upon premature death from firearm injuries, the costs of gun violence was estimated to be $\$ 229$ billion in 2012 [9]. To put that amount in perspective, the costs of gun violence was $\$ 88$ billion more than the US Federal Government budgeted for education that year, costing every man, woman, and child in the US over $\$ 700$ a person.

In addition to the more direct costs related to gun violence, homicides and gun shootings also have a negative impact upon home values and business success in the communities where they take place. For example, in Minneapolis, each additional gun homicide in a census tract was associated with a $\$ 22,000$ decline in average home values and 80 fewer jobs the following year [10].

\section{Effectiveness of Gun Control Laws}

Gun control laws have had limited success in reducing US gun related mortality. From 1999 to 2013, the firearm fatality rate in the US has remained unchanged [8]. Furthermore, when looking at 25 different gun control laws implemented by states in the US, nine were found to be beneficial, nine found to be counterproductive, and seven were inconclusive [4].

This is not to say that gun control laws have no effect. Although the overall firearm fatality rate in the US has not changed since 1999, some individual states have had more success in limited firearm related deaths. In general, states with more restrictive gun laws have a lower firearm fatality rate [8]. Also, it has been shown that if the top three most effective gun control laws were uniformly applied to everyone in the US, firearm mortality rates could be reduced from 10.35 per 100,000 down to less than 1 per 100,000. These three gun control laws thought to be most effective are universal background checks for the purchase of guns, background checks for the purchase of ammunition, and firearm identification through ballistic fingerprinting or microstamping [4].

\section{Blockchain Applications in Gun Control}

Blockchain applications in gun control are most likely to be effective if they help facilitate background checks, help track the flow of guns, and help create a stable economic climate. Blockchain protocol can help with background checks by helping create and maintain a database that is accurate, resistant to hacking, and easy to access. Tracking the transfer of guns from manufacturer to dealer to end user can also be readily improved through blockchain technology, similar to what is being done in other markets that require accurate tracking to ensure safety. Finally, blockchain applications in central banking have the potential to allow finer tuning of the economy and a more rapid response to economic recessions.

Background checks currently are typically performed by querying the National Instant Criminal Background Check System (NICS). The NICS is a series of databases maintained by the Federal Bureau of Investigation (FBI). If a query turns up negative, then the gun transfer is approved typically within minutes. However, if 
the person desiring to purchase the gun shows up on the database, the law allows for three days to further investigate prior to allowing the sale.

The NICS system is a centralized, hub and spoke type of database. All inquiries must be made to the central database which is maintained by a single organization. This type of database structure has a low fault tolerance in that it presents a single point of attack for a hacker and a single point for human errors in data entry and management. The imperfect nature of the NCIS is even recognized by the FBI, which reports that about 3000 people pass the NICS background check every year, in spite of being prohibited from buying a gun by state or federal law [11]. Fully distributed databases, such as the distributed bitcoin ledger, are more robust against attack, are easier to update, easier to maintain, and could also track the flow of guns from manufacturer to end user.

The strength of the blockchain protocol in maintaining an accurate ledger is widely recognized as a significant improvement in product tracking. For example, Walmart has partnered with IBM to create a blockchain system of tracking food and supply chains [12]. Blockchain systems are also being proposed as a better way to control nuclear energy exports and control the distribution of marijuana [13, 14].

Blockchain applications in finance could greatly improve economic stability globally, and as a result have a positive impact upon public health and gun violence by decreasing the conditions that potentially increase violence such as high unemployment and business recessions. Cryptocurrencies, which use the blockchain protocol, could potentially help stabilize central banks by boosting their reserves or by decreasing their seigniorage [15]. Although central banks do not have uniform policies regarding cryptocurrencies, there is a growing realization that bitcoin and other cryptocurrencies are here to stay $[16,17]$. In order to maximize public health benefits of blockchain in finance, an aggressive approach at integrating the technology right now into existing systems is recommended [18].

\section{Implementation}

A blockchain solution to gun control would work similar to current cryptocurrency implementations of blockchain technology. First, individuals currently owning a gun or purchasing a gun would get an electronic gun safe, similar to a bitcoin (BTC) wallet. This wallet would ideally be tied to biometric data such as a retina scan or fingerprint. Whenever a gun was created, purchased or sold, the transaction from one electronic gun safe to another would be recorded on the blockchain in an immutable, time-stamped manner.

All transactions would require both the seller and a licensed gun dealer to approve the sale. Similar to a multi-sig wallet, both parties would need to sign the transfer. Similarly, prior to the transfer, the receiver of the gun would have to pass a background check. Until this was done, ownership could not be transferred. This would operate similar to an Ethereum based smart contract. If they pass the background check, the transfer is allowed, and if not, the transfer is denied and recorded in that individual's electronic gun safe.

Ideally, the contents of the electronic gun safe would not only include the gun's identification by ballistic fingerprinting or microstamping, it would also include pertinent data about that individual. For example, a person's electronic gun safe could include a wide range of data such as history of illegal activity, parole status, domestic violence convictions, and mental health issues. This data could be embedded in the electronic gun safe in a manner much more private than existing databases are able to achieve. Although privacy concerns may prohibit additional data being placed in the electronic gun safe, some countries may find it acceptable to also embed data from Internet browsing, similar to marketing data Facebook, Google, and Amazon currently collect. Artificial intelligence methods similar to those used in Internet marketing could potentially identify relevant mental health issues, such as an obsession with gun violence as found, posthumously, in a recent school shooter $[19,20]$. A person's browsing information is already being extensively tracked commercially, and this information could be incorporated into the electronic gun safe. The No-Fly List could be incorporated into the blockchain [21]. An obsession with guns or gun violence may not automatically trigger a denial of gun ownership, but it could at least trigger a more thorough investigation of the potential buyer prior to the sale being finalized. Total number of guns owned could be more reliably tracked. This 
could potentially identify high risk individuals such as the Las Vegas shooter, who bought 33 guns in the 12 months preceding his mass shooting [22].

Implementing blockchain technology into gun control is both necessary from a public health standpoint and is economically viable. Simply creating a blockchain record of transactions would save an enormous amount of money by decreasing the amount of labor required to perform gun traces. Implementation would be straightforward, as the underlying technology is already well developed. Individuals would have their own, unique electronic gun safe which would operate similar to a BTC wallet. Gun transactions would be similar to spending and receiving bitcoins. Using multisig wallet and smart contract technology, the safe transfer of guns between legal owners satisfying current laws could be reliably performed. Privacy concerns could be achieved using anonymous technology that has already been implemented in ZCash, Monero, and DASH. Implementing better technology, not just more laws, is key to achieving better gun control and reducing gun related violence.

Through the use of colored coins, the proposal laid out above could be built on top of the established bitcoin blockchain. Colored coins bind meta-data to a specific bitcoin transaction, effectively turning the bitcoin into a token by "coloring" it with the meta-data. This meta-data can contain information on assets such as guns, and additional data describing the gun owner [23].

Finally, putting background check information, gun transfer information, and gun owner information onto a blockchain system, there would be a much greater ability to determine the overall gun ownership in the US. A gun regulatory board could then be setup to help determine and balance the legal rights to gun ownership with the public health concerns of excessive gun availability. This gun regulatory board could thus operate very loosely like a central bank, limiting the importation or manufacture of new guns when the overall number of guns in society exceeded a certain level. Although gun ownership is a constitutional right in the US, guns also are the agent, the vector if you will, of an established public health epidemic. Tracking guns with advanced blockchain protocols will to help determine overall gun burden upon society, so reasonable gun control measures can be implemented.

\section{Conclusion}

Effective gun control in the US requires the the number of guns available in society be limited in ways that are compatible with both the constitution and public health. The blockchain protocol is the most accurate system to track gun flow from manufacturer to end user, and track sales from one gun owner to another.

It allows for better privacy than existing background check systems, and simultaneously link ownership of a specific gun to a specific individual in an immutable manner. A blockchain based digital gun safe would improve the implementation of existing gun laws, maintaining privacy and improving gun control. Gun control should not be viewed in isolation as a legal issue that can be solved simply by the creation of more laws. Blockchain technology, when applied to the financial systems and businesses, can potentially help create a climate where the rates of suicide and interpersonal violence are minimized.

\section{Bibliography}

1. Kochanek KD, Murphy SL, Xu J, Tejada-Vera B (2016) Deaths: final data for 2014. Natl Vital Stat Rep 65:1-122.

2. Bauchner H, Rivara FP, Bonow RO, et al. (2017) Death by Gun Violence-A Public Health Crisis. JAMA. doi: $10.1001 /$ jama.2017.16446

3. Bill of Rights. In: Bill of Rights Institute. https://www.billofrightsinstitute.org/foundingdocuments/bill-of-rights/. Accessed 16 Oct 2017

4. Kalesan B, Mobily ME, Keiser O, et al. (2016) Firearm legislation and firearm mortality in the USA: a cross-sectional, state-level study. Lancet 387:1847-1855. doi: 10.1016/S0140-6736(15)01026-0 
5. Fontaine J, Jannetta J, Papachristos A, et al. (2017) Put the Guns Down. In: Urban Institute. https: //www.urban.org/research/publication/put-guns-down. Accessed 21 Oct 2017

6. Luo F, Florence CS, Quispe-Agnoli M, et al. (2011) Impact of business cycles on US suicide rates, 1928-2007. Am J Public Health 101:1139-1146. doi: 10.2105/AJPH.2010.300010

7. WISQARS Years of Potential Life Lost (YPLL) Report, 1999 and later - NCIPC. https://webappa. cdc.gov/sasweb/ncipc/ypll.html . Accessed 23 Oct 2017

8. Resnick S, Smith RN, Beard JH, et al. (2017) Firearm deaths in america: can we learn from 462,000 lives lost? Ann Surg 266:432-440. doi: 10.1097/SLA.0000000000002376

9. Follman M, Lurie J, Lee J, West J (2015) The True Cost of Gun Violence in America. In: Mother Jones. http://www .motherjones.com/politics/2015/04/true-cost-of-gun-violence-in-america/ . Accessed 22 Oct 2017

10. Irvin-Erickson Y, Lynch M, Gurvis A, MohrBing Bai E (2017) Gun Violence Affects the Economic Health of Communities. In: Urban Institute. https://www.urban.org/research/publication/gun-violenceaffects-economic-health-communities . Accessed 22 Oct 2017

11. NICS \& Reporting Procedures. In: Giffords Law Center to Prevent Gun Violence. http://lawcenter. giffords.org/gun-laws/policy-areas/background-checks/nics-reporting-procedures/ . Accessed 22 Oct 2017

12. (2016) Walmart, IBM and Tsinghua University Explore the Use of Blockchain to Help Bring Safer Food to Dinner Tables Across China. http://www-03.ibm.com/press/us/en/pressrelease/50816.wss . Accessed 21 Oct 2017

13. Arnold A (2017) Blockchain: A new aid to nuclear export controls? In: Bulletin of the Atomic Scientists. https://thebulletin.org/blockchain-new-aid-nuclear-export-controls11204. Accessed 21 Oct 2017

14. Making Marijuana Safer: Jessica Versteeg Explains Paragon Coin, The New Cannabis Blockchain | Benzinga. https://www.benzinga.com/trading-ideas/long-ideas/17/10/10201520/making-marijuana-saferjessica-versteeg-explains-paragon-coi . Accessed 22 Oct 2017

15. Moore W, Stephen J Should Cryptocurrencies be included in the Portfolio of International Reserves held by the Central Bank of Barbados? In: Central Bank of Barbados. https://goo.gl/yCdPqD . Accessed 13 Oct 2017

16. He D, Habermeier K, Leckow R, et al. (2016) Virtual Currencies and Beyond: Initial Considerations. Staff Discussion Notes 16:1. doi: 10.5089/9781498363273.006

17. Legarde C (2017) Central Banking and Fintech - A Brave New World? In: International Monetary Fund. https://www.imf.org/en/News/Articles/2017/09/28/sp092917-central-banking-and-fintech-a-bravenew-world. Accessed 5 Oct 2017

18. Froelings L (2017) Microsoft CEO Issues Blockchain Challenge to Interbank Messaging Service Swift. https://cointelegraph.com/news/microsoft-ceo-issues-blockchain-challenge-to-interbank-messagingservice-swift. Accessed 23 Oct 2017

19. Sokol C, Glover J, Francovich E, et al. (2017) One student dead, three in hospital after classmate opens fire at Freeman High School. In: The Spokesman-Review. http://www.spokesman.com/stories/2017/ sep/13/shooting-reported-at-freeman-high-school/ . Accessed 16 Oct 2017

20. (2017) Freeman High School shooting: Suspect said he was bullied, police documents say. In: CBS News. https://www.cbsnews.com/news/freeman-high-school-shooting-suspect-said-he-was-bullied-policedocuments-say/. Accessed 16 Oct 2017 
21. (2017) Banking Is Only The Beginning: 30 Big Industries Blockchain Could Transform. In: CB Insights. https://www.cbinsights.com/research/industries-disrupted-blockchain/. Accessed 16 Oct 2017

22. Johnson K, Jervis R (2017) Las Vegas shooting: Stephen Paddock bought 33 guns in last 12 months. In: USA Today. https://www . usatoday.com/story/news/nation/2017/10/04/las-vegas-shooter-bought33-guns-last-12-months/730634001/ . Accessed 16 Oct 2017

23. Rosenfeld M (2012) Overview of Colored Coins. https://bitcoil.co.il/BitcoinX.pdf . Accessed 11 Nov 2017 